\section{Lebensjahre in Krankheit nehmen zu}

Tetzlaff J et al. Expansion or compression of multimorbidity? 10-year development of life years spent in multimorbidity based on health insurance claims data of Lower Saxony,

Germany. Int J Publ Health 2017; 62: 679-686

Chronische Erkrankungen werden mit zunehmendem Alter immer häufiger. Wenn immer mehr Menschen ein hohes und sehr hohes Lebensalter erreichen, liegt der Verdacht nahe, dass die Zahl der Lebensjahre mit Multimorbidität ebenfalls ansteigt. Eine Studie von Juliane Tetzlaff von der Medizinischen Hochschule Hannover und Koautoren untersuchte die Entwicklung der Lebensjahre mit und ohne Multimorbidität von Senioren in Niedersachsen.

Die Analyse der Trends über 10 Jahre hinweg basiert auf Daten von Versicherten der AOK Niedersachsen im Alter von 60 Jahren und älter aus den Jahren 2005 2014. Multimorbidität wurde in der Studie definiert als die Kombination von mindestens 6 chronischen Erkrankungen und einer Polypharmazie (mindesten 5 verschiedene Verordnungen innerhalb eines Jahres). Die Autoren ermittelten zunächst die altersstandardisierte Prävalenz von Multimorbidität, um anschließend die Entwicklung der Zahl der Lebensjahre mit und ohne Multimorbidität und den Anteil der Lebensjahre mit Multimorbidität an allen Lebensjahren (Morbiditätsverhältnis) abschätzen zu können. Die Lebenserwartung eines 60-jährigen Mannes betrug in Niedersachsen im Jahr 2005 19,18 Jahre, die der 60-jährigen Frauen 24,03 Jahre. Bis 2014 stieg die Lebenserwartungen der 60-Jährigen um 0,69 Jahre bei den Männern und 0,63 Jahre bei den Frauen an. Der Anteil

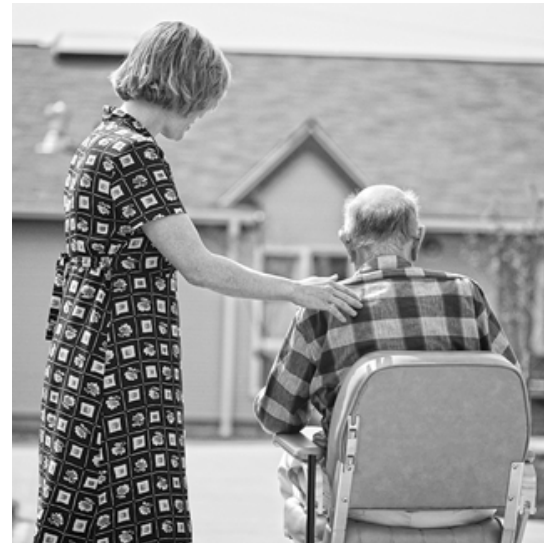

- Der Anteil der Lebensjahre, die ältere Menschen in Multimorbidität verbringen, steigt: Männer im Alter von 60 Jahren im Jahr 2014 mussten damit rechnen, gut 6 Jahre ihrer verbleibenden Lebenszeit multimorbid zu sein, Frauen sogar 8 Jahre. Bildquelle: PhotoDisc (Symbolbild)

der Personen mit Multimorbidität stieg dabei ebenfalls an: 2005 wiesen 18,6\% der über 60 Jahre alten Männer und $22,1 \%$ der entsprechend alten Frauen eine Multimorbidität auf, 2014 lag der Anteil mit 31,6\% bei Männern und 35,0\% bei Frauen jeweils um etwa $13 \%$ höher. Der Anteil der Lebensjahre, die in Multimorbidität verbracht wurden, stieg im Beobachtungszeitraum um 2,7 Jahre bei Männern und 3,2 Jahre bei Frauen an. Damit mussten Männer im Alter von 60 Jahren im Jahr 2014 damit rechnen, 6,1 Jahre ihrer verbleibenden Lebenszeit multimorbid zu sein, Frauen sogar 8 Jahre. Die Jahre ohne Multimorbidität haben bei insgesamt gestiegener Lebenserwartung und mehr Jahren mit Multimorbidität im Beobachtungszeitraum abgenommen. Weitere Auswertungen der Daten, bspw. im Hinblick auf Entwicklungen in unterschiedlichen sozioökonomischen Gruppen oder hinsichtlich der Zusammenhänge von Multimorbidität und Behinderung/Abhängigkeit sind geplant.

\section{FAZIT}

Die Autoren fanden einen klaren Beleg für eine Expansion der Jahre mit Multimorbidität parallel zur steigenden Lebenserwartung. Dies führt zu wachsenden Belastungen der Individuen wie auch des Gesundheitssystems. Die Befunde weisen auf die große Bedeutung von Prävention und gesundem Lebensstil über die gesamte Lebensspanne hin. Die Autoren empfehlen zudem die Entwicklung von Versorgungsstrategien, die spezifisch auf die Bedarfe der alten multimorbiden Patienten ausgerichtet sind.

Friederike Klein, München 